\title{
Inducing immune tolerance in RA: clinical and immunologic efficacy
}

Epitope-specific immunotherapy is based on the principle of reducing the reactivity of the adaptive immune system to proinflammatory epitopes by inducing immune tolerization. A pilot phase II trial conducted by Koffeman and colleagues tested the immunologic and clinical efficacy of administering the tolerogen DNAJP1 (a dominant epitope of the heat shock protein DNAJ) to patients with active RA.

All 160 patients included in the study had immunologic sensitivity to DNAJP1 at baseline. Participants received either oral DNAJP1 (25 mg daily) or placebo for 6 months.

After 168 days' treatment, the percentage of T cells producing tumor necrosis factor (TNF) was considerably reduced from baseline in DNAJP1-

treated patients compared with those who received placebo, indicating the induction of an immune deviation by the tolerogen.
The percentage of patients who achieved the American College of Rheumatology criteria for $20 \%$ improvement tended to be higher in the DNAJP1 group than in the placebo group at the study time points. This difference was significant in the subgroup of patients who were receiving concomitant hydroxychloroquine therapy, suggesting a possible synergy between this drug and DNAJP1.

The authors also noted that clinical response was dependent on elevated expression of several molecules associated with downregulation of adaptive immunity at baseline. Identification of biomarkers that predict response to this treatment is warranted in further studies.

Nick Warde

Original article Koffeman, E. C. et al. Epitope-specific immunotherapy of rheumatoid arthritis. Arthritis Rheum. 60, 3207-3216 (2009) 